\title{
Comment on 2018 American Academy of Neurology guidelines on disease-modifying therapies in MS
}

John R. Corboy, MD, Brian G. Weinshenker, MD, and Dean M. Wingerchuk, MD

Neurology ${ }^{\circledR}$ 2018;90:1106-1112. doi:10.1212/WNL.0000000000005574

\section{Abstract}

The American Academy of Neurology has published a comprehensive review and guidelines for the use of disease-modifying therapies (DMTs) in multiple sclerosis (MS) for the first time since 2002. These guidelines represent the work of MS experts, patients, and guideline experts and are based on their review of randomized controlled trials and observational evidence that addresses a set of prespecified questions related to starting, switching, and potentially discontinuing DMTs. Many of the recommendations address decision-making regarding the use of DMTs and incorporate the perspective of patients. Modified Delphi methods were used to establish consensus recommendations that were assigned a level of clinical obligation (actions a clinician must $[\mathrm{A}]$, should $[\mathrm{B}]$, or may $[\mathrm{C}]$ do). Most guideline recommendations are level B. Few reached level A, and several achieved only level C, primarily because of lack of evidence. The guidelines eschew formal treatment algorithms and do not address financial considerations and a variety of other controversies. We identify remaining uncertainties, the most important of which is the choice of available DMTs for the average newly diagnosed patient. We reiterate a number of research needs identified in the guidelines that could affect the use of DMTs, including improved definition of breakthrough disease requiring change in therapy, development of better and universally accepted definitions of both benign and aggressive MS, more and longer-duration comparative effectiveness trials, discovery and validation of biomarkers of disease activity and response to therapy, and development of treatment strategies focused on neuroprotection, remyelination, and neural repair.
Correspondence

Dr. Corboy

john.corboy@ucdenver.edu

\section{RELATED ARTICLE}

Editorial

Complexity of MS

management in the current treatment era

Page 761

\section{Articles}

Practice guideline recommendations summary: Diseasemodifying therapies for adults with multiple sclerosis: Report of the Guideline Development, Dissemination, and Implementation Subcommittee of the American Academy of Neurology

Page 777

Comprehensive systematic review summary: Diseasemodifying therapies for adults with multiple sclerosis: Report of the Guideline Development, Dissemination, and Implementation Subcommittee of the American Academy of Neurology

Page 789 


\section{Glossary}

AAN = American Academy of Neurology; AE = adverse event; CARE-MS = Comparison of Alemtuzumab and Rebif Efficacy in Multiple Sclerosis; CIS = clinically isolated syndrome; DMT = disease-modifying therapy; EAN = European Academy of Neurology; ECTRIMS = European Committee for Treatment and Research in Multiple Sclerosis; JC virus = John Cunningham virus; MS = multiple sclerosis; NEDA = no evidence of disease activity; PPMS = primary progressive multiple sclerosis; RA = rheumatoid arthritis; RCT = randomized controlled trial; RRMS = relapsing-remitting multiple sclerosis; SPMS = secondary progressive multiple sclerosis.

Since the introduction of interferon $\beta-1 b$ as a diseasemodifying therapy (DMT) for multiple sclerosis (MS) in 1993, 15 additional DMTs have been approved by international regulatory agencies; others are used "off-label." The nature, mechanism of action, route of administration, side effects, long-term risks, efficacy, and cost of these DMTs vary considerably. When, which, and in what order DMTs should be used remains undefined. Thus, the new American Academy of Neurology (AAN) guideline for MS DMT use, ${ }^{1}$ the first since 2002, is highly anticipated.

\section{Guideline process and contents}

A panel of MS experts, patients, and guidelines experts developed recommendations for starting, switching, and stopping DMTs in patients with clinically isolated syndrome (CIS, or first clinical event), and relapsing and progressive forms of MS. The panel made no suggestions for those with radiologic features consistent with MS without clinical symptoms and signs of MS, so-called radiologically isolated syndrome. ${ }^{2}$ The panel began by systematically reviewing available evidence, mainly derived from randomized, placebo- or active comparator-controlled trials (RCTs) that addressed prespecified questions related to efficacy and harm of MS DMTs. The systematic review examined data related to efficacy (RCTs only) and harm (RCTs and observational studies). ${ }^{3}$ The review summarizes evidence for DMT efficacy compared with placebo in CIS (for the outcome of conversion to clinically definite MS), relapsing-remitting MS (RRMS) (annualized relapse rate and risk of relapse at 2 years, new MRI disease activity, and in-study disease progression), and progressive MS (relapse rate and in-study progression). For patients with disease activity on DMT, evidence for superiority of switching to a different DMT compared with no DMT change was assessed for relapse rate and MRI T2 or gadolinium-enhancing lesion activity outcomes. Risks of harm were assessed using adverse events (AEs) that required DMT discontinuation and serious or life-threatening AEs. In accordance with AAN guideline development processes, ${ }^{4}$ data from the systematic review were integrated with additional evidence not captured by the review, principles of care, and inferences derived from the available evidence. Prior to analyzing the collected data, panel members contributed to an anonymous poll to determine the "minimal clinically meaningful difference" for estimates of efficacy and AEs. Modified Delphi methods were used to establish consensus recommendations that were assigned a level of clinical obligation (actions a clinician must $[\mathrm{A}]$, should $[\mathrm{B}]$, or may [C] do).

The guideline introduces notable new statements on disease stratification (identifying and treating "highly active" MS with alemtuzumab, natalizumab, or fingolimod), disease monitoring and switching DMTs for treatment-adherent patients who experience " $\ldots 1$ or more relapses, 2 or more unequivocally new MRI lesions, or increased disability on examination, over a 1-year period" (effectively a definition of treatment failure), stopping DMTs because of an unfavorable benefit/risk profile, and offering ocrelizumab for primary progressive MS (PPMS). Most of the guideline recommendations are derived from evidence unrelated to the systematic review and emphasize patient counseling about treatment options, risks, adherence, disease and side effect monitoring, and pregnancy; these will be useful guides for achieving measures of quality MS care. Almost all guideline recommendations are level B; the few that reached level A status included incorporation of patient preferences into treatment decisions, having ongoing patient-provider dialog about treatment options during the disease course, and counseling about the increased risk of relapse after natalizumab discontinuation. Predictably, level C recommendations, often with lack of panel consensus, applied to issues with inadequate evidence or ongoing controversy, such as deferral of DMT in patients without recent inflammatory disease activity, use of natalizumab in JC virusseropositive patients, stopping therapy for apparent futility in secondary progressive MS (SPMS), and use of unapproved drugs, such as azathioprine, when approved DMTs are not accessible.

\section{Guideline context}

Although the panel utilized many areas of evidence and inference beyond the systematic review data, several clinically relevant areas were not addressed. Efficacy analyses were limited to 2-year relapse and Expanded Disability Status Scale progression outcomes and did not consider longer-duration MRI studies ${ }^{5,6}$ or other now commonplace composite outcomes such as "no evidence of disease activity" (NEDA), ${ }^{7}$ which combines clinical and MRI measures. Extensive RCT data on DMT effects on measures of brain atrophy, the strongest MRI correlate with clinical progression, ${ }^{6,8}$ were not 
considered. Observational comparative efficacy studies that assess the effects of switching to one highly active DMT or another were not reviewed. ${ }^{9}$ The current status of autologous stem cell transplantation protocols was not assessed, ${ }^{10}$ presumably because all but one of these studies have been open-label and uncontrolled. The review did not consider patient-reported outcomes such as quality of life ${ }^{11}$ or fatigue. Cost and accessibility were only superficially considered. Use of unapproved drugs from the same class (e.g., using rituximab in place of ocrelizumab) ${ }^{12}$ or biosimilar/generic preparations was not discussed, even though there are now 2 approved generic versions of glatiramer acetate.

Clinicians, patients and their advocates, and administrative bodies that govern drug payment plans will scrutinize the panel recommendations. They will find neither specific advice on starting, switching, or stopping individual drugs nor an algorithm that serves to individualize patient care based on recent disease activity, age, MRI burden of disease, comorbidities, or other factors known to affect disease progression. The lack of an explicit statement advocating for patients and physicians to be the decision-makers for when to start, stop, or discontinue individual DMTs will not deter insurance companies from "step editing" (requiring failure of one or more typically older and less efficacious DMTs before allowing access to highefficacy DMTs, based primarily on financial considerations). The results of the systematic review and the panel recommendations do, however, identify contemporary evidence gaps and outline the imperative questions for future research. These questions include initial treatment strategies (induction vs escalation approaches), the validity of composite "treat to target" outcome measures, treatment of progressive MS, and establishing the presence and magnitude of long-term benefits from drugs approved on the basis of short-term treatment (see below). While not part of this guideline, the AAN has already stated an explicit position that the patient, with assistance from their provider, should be making these complex decisions about starting, stopping, and discontinuing DMTs. ${ }^{13}$

Comparison of the new AAN recommendations to other MS DMT consensus statements is informative. The "Brain Health: Time Matters in Multiple Sclerosis" report, ${ }^{14}$ endorsed by 17 international MS groups, presented a widely held view of how MS negatively affects patients, families, and society, and emphasized the importance of rapid diagnosis and effective treatment to maximize lifelong brain health. The report supported the concept that MRI measures, including accelerated brain atrophy, should be interpreted as treatment-modifiable disease activity. The report contends that newer DMTs are more effective than older ones but does not recommend specific "first-line" treatments. It discourages DMT discontinuation, advising to "maintain treatment with a diseasemodifying therapy for as long as a person with MS would be at risk of inflammatory disease activity if they were not receiving treatment." Similarly, the consensus paper authored by the MS Coalition ${ }^{15}$ summarized current evidence about DMT use and "provide(s) support for broad and sustained access to MS disease-modifying therapies for people with MS in the United States." Neither paper strived for balance on controversial topics, such as the significance of brain atrophy or the superiority of newer DMTs. Both emphasized challenges of medication access and advocated for early and persistent intervention with MS DMTs. The new AAN guideline is comparatively more conservative and balanced while offering specific guidance about DMT use. The 2015 American College of Rheumatology Guideline for the Treatment of Rheumatoid Arthritis (RA) ${ }^{16}$ offers a systematic review of disease-modifying antirheumatic drugs and promotes algorithmic approaches to treatment of early RA and established RA, a chronic inflammatory disease with many therapeutic challenges in common with MS. The American College of Rheumatology guideline offers concrete suggestions for how to deal with high-risk patients and vaccinations. In contrast, the AAN guideline eschews specific algorithms and expressly identifies a number of instances of lack of consensus among the panel members. Finally, the European Committee for Treatment and Research in Multiple Sclerosis (ECTRIMS) and the European Academy of Neurology (EAN) developed a guideline with 20 statements on starting and switching therapies in CIS, RRMS, and progressive MS, with a significant focus on monitoring with MRI. ${ }^{17}$ The ECTRIMS/EAN guidelines steer clear of specific treatment algorithms and do not address radiologically isolated syndrome similar to the AAN guidelines. The ECTRIMS/EAN guidelines offer a rather conservative approach to treatment and do not recommend treating aggressive disease with more efficacious therapies, and recommend "Consider continuing a diseasemodifying drug if the patient is stable (clinically and on MRI) and shows no safety or tolerability issues," rather than advocating long-term use to maintain disease suppression. As with the AAN guideline, the ECTRIMS/EAN guidelines do not mention stem cell transplants, biosimilar/generics, or advocate stabilizing brain atrophy as a goal of treatment. The ECTRIMS/EAN guidelines suggest mitoxantrone as an option for active SPMS despite its rare contemporary usage and recommend ocrelizumab and cladribine for SPMS despite absence of RCTs. Finally, they do not discuss JC virus antibody serology and antibody index to guide treatment.

\section{Controversies}

\section{High-efficacy therapy at the outset}

A number of controversies remain unanswered in this guideline, primarily because of lack of data, the most important of which is the decision as to which DMT, or group of DMTs, should be used after diagnosis of relapsing MS. In the United States, insurance companies "step edit" medication choices. Many clinicians, based on some evidence, agree that newly diagnosed patients with MS who have clinical and radiographic markers of poor prognosis in the early stages of MS should be treated with agents with higher efficacy from the outset, even if associated with greater risks. Although there is general agreement about the nature of clinical, demographic, 
and other prognostic variables, how to use those variables to generate an accurate prognosis remains elusive. Indeed, the utility of presently available prognostic markers in individual patients is limited. MRI T2 lesion load at presentation has only modest predictive value. Gadolinium-enhancing lesion number and spinal cord lesions may offer more short-term prognostic information, but long-term validation of these measures is not yet available, ${ }^{18}$ and some concern has been raised about potential risk of gadolinium given evidence of long-term persistence in the brain. Thus, the lack of excellent long-term markers limits the ability to define optimal riskbenefit profiles early in the disease course. ${ }^{5,19}$ Similarly, there was lack of panel consensus for what constitutes "very active MS," a situation in which most clinicians agree that highefficacy treatments are appropriate. The guidelines suggest that patients with established "benign MS," presumably those patients who have established MS for 5 or more years but have a normal or mildly abnormal examination and a low MRI lesion burden, although there is no consensus on definition, may be appropriately not treated with DMT while monitoring disease activity. But what of the "average" newly diagnosed patient? Comparative studies of higher- vs lower-efficacy DMTs from disease onset are limited and do not include longterm follow-up. Further confusing the issue is that changing diagnostic criteria may be favorably influencing the long-term prognosis of MS, as patients with milder forms of MS are identified simply with additional MRI activity over time. Whether this reflects an earlier time point at which MS is actually diagnosed and potentially treated, or represents a milder variation of MS that perhaps does not need to be treated at all, remains an unresolved issue.

The arguments for treating the "average" newly diagnosed patient with MS using injectable medications approved more than 20 years ago and escalating to more effective but somewhat more risky therapy only if the patient has signs of significant recurrent disease activity are as follows: (1) the natural history of MS is variable and several studies suggest that as many as $40 \%{ }^{20}$ will have a benign course at 10 years from onset, although the proportion who are still benign after 20 years is $10 \%$ to $20 \%{ }^{21,22}$; (2) the significance of brain atrophy is unclear in the long term and many confounders render it unreliable as a prognostic indicator in individual cases $^{6,23,24}$; (3) a significant proportion of patients are attack-free and do well on interferon $\beta$ preparations or glatiramer acetate, and these drugs are significantly safer than more aggressive treatments ${ }^{25}$; (4) clinical and radiologic monitoring for MS disease activity (clinical relapses or new contrast-enhancing or T2 lesions), although imperfect, allows adequate identification of patients whose treatment is deemed to have failed ${ }^{26-28}$ and would benefit from treatment escalation; and (5) the relative efficacy of treatments is incompletely understood and methods to identify relative efficacy often rely on retrospective analysis and partially effective methods to control for indication and attrition bias such as propensity matching and pairwise censoring ${ }^{29}$; it is difficult to identify whether a patient will be a good responder to any particular treatment or benefit from switching treatment.

The arguments that the "average" patient should be treated aggressively from the outset, assuming appropriate risk factors such as seropositivity for JC virus are taken into account, are as follows: (1) MS has likely been active and caused brain injury for many years before becoming clinically manifest ${ }^{30}$; (2) the vast majority of patients will develop significant disability over time and important disability accrues even in "benign" patients when cognitive impairment and decreased employability are considered ${ }^{31,32}$; (3) failure to suppress disease activity in the early years after diagnosis predisposes to a secondary progressive phase of the illness and later advanced disability, ${ }^{33}$ and early disease markers that identify those who will be classified as having a "benign" course after 10 or more years are imperfect ${ }^{22}$; (4) new inflammatory disease activity that responds to presently available DMTs is most prominent early in the course of MS and the potential for effective intervention decreases over time $\mathrm{e}^{34,35}$; (5) RCT subgroup analyses have consistently shown greatest benefit in younger patients $^{36}$; (6) age-related comorbidities (e.g., diabetes, hypertension, cancer, JC virus-positive status) limit use of some DMTs later in the disease course ${ }^{37}$; and (7) despite imperfect and short-term comparison trials, newer agents, especially the monoclonal antibodies such as natalizumab, alemtuzumab, and ocrelizumab, have consistently been found to be superior in efficacy compared to interferons and glatiramer acetate. ${ }^{38}$ While it is methodologically difficult to obtain comprehensive long-term evidence for superiority of one treatment over another, a study funded by the Patient-Centered Outcomes Research Institute will address this issue directly. ${ }^{39}$

\section{Discontinuation of DMTs}

Whether and when to discontinue DMTs in stable patients are highly contentious issues with no high-quality evidence to guide decision-making. Independent of treatment, inflammatory events, such as relapses ${ }^{35}$ and new gadoliniumenhancing lesions, ${ }^{34}$ diminish in frequency with time and disease duration, consistent with low frequency of active inflammatory white matter MS lesions at autopsy of patients with long-standing MS. ${ }^{40}$ Discontinuation or interruption of DMT in patients with recently active disease often results in disease reactivation, if not rebound, as reported in patients who discontinue natalizumab ${ }^{41}$ or fingolimod. ${ }^{42}$ The guideline suggests considering DMT discontinuation in some patients with CIS or early RRMS who are doing well. This is somewhat counterintuitive, as stabilization is the main goal of therapy. However, patients with a favorable prognostic profile, or whose disease may have become quiescent with increasing age, might be reasonably observed off treatment and monitored for recurrent disease activity. This approach potentially may permit discontinuation of expensive, intrusive, and possibly unnecessary treatments. The guideline did not identify subgroups appropriate for such a strategy based on age; discontinuation in younger patients poses greater concerns given the higher chance of recurrent disease activity. A critical area 
of future research is improved determination of when inflammatory disease activity has entirely or largely ceased, ideally with a reliable biomarker. The Discontinuation of Disease Modifying Therapies in MS (NCT 03073603), ${ }^{43}$ an RCT of DMT discontinuation, funded by the PatientCentered Outcomes Research Institute and the National Multiple Sclerosis Society, will assess the effects of discontinuation in those 55 years and older with no evidence of relapse or MRI-determined disease activity for at least 5 years before enrollment.

\section{Appropriate and actionable MRI targets for "treat to target" approaches}

In other relapsing inflammatory diseases, such as RA, early potent therapies are now recommended for patients ${ }^{16}$ with unfavorable prognostic profiles as determined by regular monitoring with composite clinical, radiologic, and laboratory indicators of disease activity. A similar approach of regular monitoring and attempting to achieve NEDA based on assessment of clinical relapses, change in neurologic examination, and MRI monitoring of disease activity has recently been touted as a way of optimizing MS outcomes. ${ }^{44}$ However, the rate at which NEDA is achievable in standard outpatient practice, at least using injectable therapies as first-line therapies, even at highly specialized MS centers, is less than $10 \%$ over 7 years. ${ }^{45}$ In addition, some studies suggest that NEDA attainment is not even highly predictive of future outcome. ${ }^{46}$ Brain atrophy, especially gray matter atrophy, has been reported to correlate with disability progression, ${ }^{6}$ but the means to measure it reliably in practice are lacking, and there are a number of confounders that influence its measurement. ${ }^{23,24}$ Nevertheless, the MS community has clearly moved beyond initiation of a DMT as a standalone strategy and toward a standard of monitoring success of treatment and developing algorithms for switching when necessary to more aggressive treatments. A systematic review showed that detection of gadolinium-enhancing lesions was associated with greater predictive power for interferon treatment failure compared with detection of new T2 lesions. However, enhancing lesions may be more accurately detected and development of some T2 lesions might occur before full treatment effect has been achieved. ${ }^{26}$ A more recent analysis showed that a clinical relapse plus 3 or more new T2 lesions during the first year of therapy was associated with both higher rates of treatment failure and disability worsening. ${ }^{27}$ The level of acceptable disease activity remains to be fully established but should include a combined clinical and MRI assessment. ${ }^{28}$

\section{Treatment for PPMS}

The ORATORIO study showed that ambulatory decline was delayed in patients with PPMS treated with ocrelizumab compared to placebo (hazard ratio for 6-month confirmed worsening of disability: $0.76[0.58,0.98 ; p=0.0321]){ }^{47}$ The committee recommends that ambulatory patients should be prescribed ocrelizumab barring any contraindication or situation whereby risk outweighs benefit. This recommendation failed to meet consensus, although the final decision was to designate treatment of PPMS as a level B recommendation.
Although ocrelizumab was the first DMT to demonstrate efficacy for this disease phenotype in which many other drugs had failed, the mechanism of action of ocrelizumab is believed to be reduction of inflammatory processes in MS, which typically diminish in importance over time. Subgroup analysis of the ORATORIO study showed that the benefits were greatest in younger patients, ${ }^{48}$ especially those with evidence of ongoing inflammatory disease, in parallel to observations from other subgroup analyses in SPMS. ${ }^{49}$ Given the generally slow evolution of disability in PPMS, the observed degree of benefit is unlikely to be perceptible to individual patients. Although the committee acknowledges in its recommendations for future research that treatments need to be individualized to patient profiles, it did not recommend that clinicians select patients who are most likely to benefit, presumably patients with earlier disease and more evident disease activity on MRI.

\section{Long-term benefits from short-term treatment in patients with early MS}

The alemtuzumab clinical trials targeted patients in early phases of MS, either treatment naive (CARE-MS I $)^{50}$ or having failed one or more treatments (CARE-MS II), ${ }^{51}$ to address the hypothesis that early treatment with aggressive therapy might be more efficacious than later treatment. Alemtuzumab was more effective than thrice weekly subcutaneous interferon $\beta$-1a. Follow-up studies suggest that patients treated with alemtuzumab have high rates of long-term remission despite treatment with yearly induction therapy for only 2 or 3 years. ${ }^{52}$ However, there was no convincing difference in efficacy between patients treated with alemtuzumab in CARE-MS I vs those who were treated in CARE-MS II, and a comparative group that was treated in later phases of MS was lacking. Nevertheless, the long-term alemtuzumab findings are consistent with the observation that short-term (2-year) treatment with cladribine, ${ }^{53}$ another treatment regarded as quite aggressive, provided robust and lasting benefits for highly active MS. The European Medicines Agency recently approved cladribine on the basis of these post hoc analyses, ${ }^{54}$ and it appears that approval will be sought in the United States as well.

\section{Future needs}

The AAN committee has presented a useful list of future requirements, including improved disease outcomes reflecting all relevant outcomes; definition of breakthrough disease and acceptable level of disease activity; better defining of both benign and highly active MS and matching intensity and aggressiveness of treatment to these definitions; improving strategies to determine comparative effectiveness; and defining when treatment can be stopped. Decisions about when to initiate treatment and how aggressively and how long to treat are ideally informed not only by evidence of short-term efficacy from clinical trials but also by evidence of long-term benefit on robust measures, such as large increments of disability of unassailable significance. ${ }^{55}$ These treatment decisions should be based on prediction of treatment response in 
individual patients, aiming for precision and personalized medicine in MS care. ${ }^{56}$ Data on this subject are generally lacking, and long-term outcome studies are often confounded by biased decisions about continuation of treatment and biased follow-up. ${ }^{57}$ Results have generally, but not universally, supported long-term benefits, and study discordance might be explained by biases inherent in physician decisions to initiate immunomodulatory treatments. For example, withholding of treatment with perceived benign prognosis and from patients with progressive MS would be expected to have opposite effects on the long-term results of treatment when compared to those who did not receive treatment. Stratification into prognostic tiers using widely accepted prognostic composites has assisted investigators in demonstrating benefits of interferon $\beta$ treatment in some "real world" studies. Comprehensive long-term follow-up of RCT participants and improved strategies of Bayesian stratification based on baseline and on-treatment "intermediate" outcomes are likely to be of increasing importance, ${ }^{57}$ as are data from large, real-world cohorts such as MSBase. ${ }^{58}$

Validated therapeutic biomarkers that are individualized to the mechanism of action of the drug and can predict treatment success would improve the cost-effectiveness of DMTs. Biomarkers should be sensitive to the mechanisms driving progression of disability, whether inflammation or neurodegeneration, the relative importance of which changes over time and with advancing disability. Inflammation, at least as detectable by new and gadolinium-enhancing MRI lesions, which are most clearly affected by current treatments, becomes less apparent over time.

Comparative studies between treatments are difficult to conduct, especially in the absence of a highly sensitive and reliable outcome measure. Although head-to-head comparative studies are the most rigorous, they require very large sample sizes to demonstrate differential efficacy utilizing current accepted but insensitive outcome measures such as attack frequency and increasing Expanded Disability Status Scale score.

While anti-inflammatory strategies are believed to be critical in early phases of the disease, early axonal protection and rapid reversal of demyelination are also likely to be important for prevention of irreversible axonal loss. Treatments that enhance repair are not yet available. Progress may be hindered by the lack of well-defined clinical, radiologic, or laboratory methodologies to assess the success of reparative treatments. However, the advent of neurofilament detection in $\mathrm{CSF}^{59}$ and potentially in serum ${ }^{60}$ are promising, as are emerging neuroimaging strategies (magnetic transfer ratio and myelin water measurements) to assess the extent of remyelination. ${ }^{61} \mathrm{Re}$ generative strategies are also important targets of treatment, and studies targeting these strategies are in their infancy.

Emerging treatment strategies not covered in this review are cell-based therapies that may have capacity to home to the site of pathology and produce a more coordinated and biologically appropriate reparative response of cytokines and growth factors than is possible with current pharmacotherapy. Remyelinationpromoting treatments that stimulate endogenous myelin repair, neutralize inhibitory influences to remyelination (opicinumab, anti-LINGO-1), ${ }^{62}$ stimulate differentiation of oligodendrocyte precursor cells, ${ }^{63}$ or directly introduce remyelinating cells are emerging as potential treatment strategies. ${ }^{64}$

The pace of introduction of new treatments and emerging AEs that require specific risk mitigation efforts and anticipated studies that inform when and how to switch or stop treatments will necessitate more frequent guideline updates. The neurology community would benefit from a standing committee that regularly updates treatment guidelines based on emerging evidence. Its recommendations might even serve as an impetus for initiation of studies that address some of these key issues.

\section{Author contributions}

Study concept and design: Drs. Corboy, Weinshenker, and Wingerchuk. Acquisition of data: Drs. Corboy, Weinshenker, and Wingerchuk. Analysis and interpretation: Drs. Corboy, Weinshenker, and Wingerchuk. Critical revision of the manuscript: Drs. Corboy, Weinshenker, and Wingerchuk. Study supervision: Drs. Corboy, Weinshenker, and Wingerchuk.

\section{Study funding}

No targeted funding reported.

\section{Disclosure}

J.R. Corboy: research funding from the National Multiple Sclerosis Society, Patient-Centered Outcomes Research Institute, MedDay, Novartis, Biogen; receives compensation as editor of Neurology: Clinical Practice; has received speaking honorarium from PRIME CME and the Rocky Mountain MS Center. B.G. Weinshenker: royalties from RSR Ltd., Oxford University, Hospices Civil de Lyon, and MVZ Labor PD Dr. Volkmann und Kollegen GbR for a patent of NMO-IgG as a diagnostic test for NMO and related disorders; member of an adjudication committee for clinical trials in NMO being conducted by MedImmune and Alexion pharmaceutical companies; consultant for Caladrius Biosciences and Brainstorm Therapeutics regarding potential clinical trials for $\mathrm{NMO}$; member of data safety monitoring committee for clinical trials in multiple sclerosis conducted by Novartis. D. M. Wingerchuk: personal compensation as a consultant for Caladrius Biosciences, Inc., Brainstorm Cell Therapeutics, and Celgene; as a member of the adjudication committee of a clinical trial for MedImmune; and as co-editor-in-chief of The Neurologist; research/grant support for clinical trials from Alexion Pharmaceuticals, Inc., and Terumo BCT, Inc. Go to Neurology.org/N for full disclosures.

Received January 19, 2018. Accepted in final form March 27, 2018.

\section{References}

1. Rae-Grant A, Day GS, Marrie RA, et al. Practice guideline recommendations summary: disease-modifying therapies for adults with multiple sclerosis. Neurology 2018; 90:777-788. 
2. Yamout B, Al Khawajah M. Radiologically isolated syndrome and multiple sclerosis. Mult Scler Relat Disord 2017;17:234-237.

3. Rae-Grant A, Day GS, Marrie RA. Comprehensive systematic review summary: diseasemodifying therapies for adults with multiple sclerosis. Neurology 2018;90:789-800.

4. AAN (American Academy of Neurology). Clinical Practice Guideline Process Manual, 2011 ed. St. Paul: American Academy of Neurology; 2011.

5. Fisniku LK, Brex PA, Altmann DR, et al. Disability and T2 MRI lesions: a 20-year follow-up of patients with relapse onset of multiple sclerosis. Brain 2008;131: 808-817.

6. Fisniku LK, Chard DT, Jackson JS, et al. Gray matter atrophy is related to long-term disability in multiple sclerosis. Ann Neurol 2008;64:247-254

7. Smith AL, Cohen JA, Hua LH. Therapeutic targets for multiple sclerosis: current treatment goals and future directions. Neurotherapeutics 2017;14:952-960.

8. Popescu V, Agosta F, Hulst HE, et al. Brain atrophy and lesion load predict long term disability in multiple sclerosis. J Neurol Neurosurg Psychiatr 2013;84:1082-1091.

9. Kalincik T, Horakova D, Spelman T, et al. Switch to natalizumab versus fingolimod in active relapsing-remitting multiple sclerosis. Ann Neurol 2015;77:425-435.

10. Scolding NJ, Pasquini M, Reingold SC, et al. Cell-based therapeutic strategies for multiple sclerosis. Brain 2017;140:2776-2796.

11. Miller D, Rudick RA, Hutchinson M. Patient-centered outcomes: translating clinical efficacy into benefits on health-related quality of life. Neurology 2010;74(suppl 3): S24-S35.

12. Kister I, Corboy JR. Reducing costs while enhancing quality of care in MS. Neurology 2016;87:1617-1622.

13. Corboy JR, Halpern J, Langer-Gould AM, Mattson DH, Schwarz H, Homonoff M. AAN position: availability of disease modifying therapies (DMT) for treatment of relapsing forms of multiple sclerosis [online]. Available at: aan.com/policy-andguidelines/policy/position-statements/availability-of-disease-modifying-therapiesdmt-for-treatment-of-relapsing-forms-of-multiple-sclerosis/. Accessed December 15, 2017.

14. Giovannoni G, Butzkueven H, Dhib-Jalbut $\mathrm{S}$, et al. Brain health: time matters in multiple sclerosis [online]. Available at: msbrainhealth.org/perch/resources/brainhealth-time-matters-in-multiple-sclerosis-sep-17-1.pdf. Accessed December 1, 2017.

15. MS Coalition. The use of disease-modifying therapies in multiple sclerosis: principles and current evidence [online]. Available at: $\mathrm{ms}$-coalition.org/cms/images/stories/ dmt_consensus_ms_coalition042017.pdf. Accessed December 1, 2017.

16. Singh JA, Saag KG, Bridges SL Jr, et al. 2015 American College of Rheumatology guideline for the treatment of rheumatoid arthritis. Arthritis Care Res 2016;68:1-25.

17. Montalban X, Gold R, Thompson AJ, et al. ECTRIMS/EAN guideline on the pharmacological treatment of people with multiple sclerosis. Mult Scler J 2018;24: 96-120.

18. Tintore M, Rovira À, Río J, et al. Defining high, medium and low impact prognostic factors for developing multiple sclerosis. Brain 2015;138:1863-1874.

19. Río J, Rovira À, Tintoré $M$, et al. Disability progression markers over 6-12 years in interferon- $\beta$-treated multiple sclerosis patients. Mult Scler 2017;24:322-330.

20. Thompson AJ, Hutchinson M, Brazil J, Feighery C, Martin EA. A clinical and laboratory study of benign multiple sclerosis. QJ Med 1986;58:69-80.

21. Pittock SJ, McClelland RL, Mayr WT, et al. Clinical implications of benign multiple sclerosis: a 20-year population-based follow-up study. Ann Neurol 2004;56:303-306.

22. Sayao AL, Devonshire V, Tremlett H. Longitudinal follow-up of "benign" multiple sclerosis at 20 years. Neurology 2007;68:496-500.

23. Bermel RA, Bakshi R. The measurement and clinical relevance of brain atrophy in multiple sclerosis. Lancet Neurol 2006;5:158-170.

24. De Stefano N, Giorgio A, Battaglini M, et al. Assessing brain atrophy rates in a large population of untreated multiple sclerosis subtypes. Neurology 2010;74:1868-1876.

25. Durelli L, Verdun E, Barbero P, et al. Every-other-day interferon beta-1b versus onceweekly interferon beta-1a for multiple sclerosis: results of a 2-year prospective randomised multicentre study (INCOMIN). Lancet 2002;359:1453-1460.

26. Dobson R, Rudick RA, Turner B, Schmierer K, Giovannoni G. Assessing treatment response to interferon- $\beta$ : is there a role for MRI? Neurology 2014;82:248-254.

27. Sormani MP, Gasperini C, Romeo M, et al. Assessing response to interferon- $\beta$ in a multicenter dataset of patients with MS. Neurology 2016;87:134-140.

28. Wattjes MP, Rovira A, Miller D, et al. Evidence-based guidelines: MAGNIMS consensus guidelines on the use of MRI in multiple sclerosis-establishing disease prognosis and monitoring patients. Nat Rev Neurol 2015;11:597-606.

29. He A, Spelman T, Jokubaitis V, et al. Comparison of switch to fingolimod or interferon beta/glatiramer acetate in active multiple sclerosis. JAMA Neurol 2015;72: $405-413$.

30. Giovannoni G. The neurodegenerative prodrome in multiple sclerosis. Lancet Neurol 2017;16:413-414

31. Ton AMM, Vasconcelos CCF, Alvarenga RMP. Benign multiple sclerosis: aspects of cognition and neuroimaging. Arq Neuropsiquiatr 2017;75:394-401.

32. Weinshenker BG, Bass B, Rice GP, et al. The natural history of multiple sclerosis: a geographically based study. I. Clinical course and disability. Brain 1989;112: 133-146.

33. Scalfari A, Neuhaus A, Degenhardt A, et al. The natural history of multiple sclerosis: a geographically based study 10: relapses and long-term disability. Brain 2010;133: 1914-1929.

34. Filippi M, Wolinsky JS, Sormani MP, Comi G; European/Canadian Glatiramer Acetate Study Group. Enhancement frequency decreases with increasing age in relapsing-remitting multiple sclerosis. Neurology 2001;56:422-423.
35. Tremlett H, Zhao Y, Joseph J, Devonshire V; UBCMS Clinic Neurologists. Relapses in multiple sclerosis are age- and time-dependent. J Neurol Neurosurg Psychiatry 2008;79:1368-1374.

36. Signori A, Schiavetti I, Gallo F, Sormani MP. Subgroups of multiple sclerosis patients with larger treatment benefits: a meta-analysis of randomized trials. Eur J Neurol 2015;22:960-966.

37. Marrie RA, Horwitz R, Cutter G, Tyry T, Campagnolo D, Vollmer T. Comorbidity delays diagnosis and increases disability at diagnosis in MS. Neurology 2009;72: $117-124$.

38. Disease-Modifying Therapies for Relapsing-Remitting and Primary-Progressive Multiple Sclerosis: Effectiveness and Value: Final Evidence Report [online]. Available at: icer-review.org/wp-content/uploads/2016/08/CTAF_MS_Final_Report_030617.pdf. Accessed December 1, 2017.

39. A Pragmatic Trial to Evaluate the Intermediate-Term Effects of Early, Aggressive Versus Escalation Therapy in People with Multiple Sclerosis [online]. Available at pcori.org/research-results/2017/pragmatic-trial-evaluate-intermediate-term-effectsearly-aggressive-versus.

40. Frischer JM, Weigand SD, Guo Y, et al. Clinical and pathological insights into the dynamic nature of the white matter multiple sclerosis plaque. Ann Neurol 2015;78 $710-721$.

41. Fagius J, Feresiadou A, Larsson EM, Burman J. Discontinuation of disease modifying treatments in middle aged multiple sclerosis patients: first line drugs vs natalizumab. Mult Scler Relat Disord 2017;12:82-87.

42. Forci B, Mariottini A, Mechi C, Massacesi L, Repice A. Disease reactivation following fingolimod withdrawal in multiple sclerosis: two case reports. Mult Scler Relat Disord 2017; 15:24-26.

43. Discontinuation of Disease Modifying Therapies (DMTs) in Multiple Sclerosis (MS) (DISCOMS) [online]. Available at: clinicaltrials.gov/ct2/show/NCT03073603? term=NCT+03073603\&rank=1. Accessed December 1, 2017.

44. Imitola J, Racke MK. Is no evidence of disease activity a realistic goal for patients with multiple sclerosis? JAMA Neurol 2015;72:145-147.

45. Rotstein DL, Healy BC, Malik MT, Chitnis T, Weiner HL. Evaluation of no evidence of disease activity in a 7-year longitudinal multiple sclerosis cohort. JAMA Neuro 2015;72:152-158.

46. University of California, San Francisco MS-EPIC Team, Cree BA, Gourraud PA, Oksenberg JR, et al. Long-term evolution of multiple sclerosis disability in the treatment era. Ann Neurol 2016;80:499-510.

47. Montalban X, Hauser SL, Kappos L, et al. Ocrelizumab versus placebo in primary progressive multiple sclerosis. N Engl J Med 2017;376:209-220.

48. Application number: 761053 Origls 000 medical review(s) [online]. Available at accessdata.fda.gov/drugsatfda_docs/nda/2017/761053orig1s000medr.pdf.

49. Kappos L, Weinshenker B, Pozzilli C, et al. Interferon beta-1b in secondary progressive MS: a combined analysis of the two trials. Neurology 2004;63:1779-1787.

50. Cohen JA, Coles AJ, Arnold DL, et al. Alemtuzumab versus interferon beta 1a as firstline treatment for patients with relapsing-remitting multiple sclerosis: a randomised controlled phase 3 trial. Lancet 2012;380:1819-1828.

51. Coles AJ, Twyman CL, Arnold DL, et al. Alemtuzumab for patients with relapsing multiple sclerosis after disease-modifying therapy: a randomised controlled phase 3 trial. Lancet 2012;380:1829-1839.

52. Havrdova E, Arnold DL, Cohen JA, et al. Alemtuzumab CARE-MS I 5-year follow-up: durable efficacy in the absence of continuous MS therapy. Neurology 2017;89: $1107-1116$

53. Giovannoni G, Comi G, Cook S, et al. A placebo-controlled trial of oral cladribine for relapsing multiple sclerosis. N Engl J Med 2010;362:416-426.

54. European Medicines Agency. Available at: ema.europa.eu/ema/index.jsp? curl=pages $/$ medicines $/$ human/medicines $/ 004230 /$ human_med_002150.jsp\& mid=WC0b01ac058001d 124

55. Wingerchuk DM, Weinshenker BG. Disease modifying therapies for relapsing multiple sclerosis. BMJ 2016;354:i3518.

56. Kalincik T, Manouchehrinia A, Sobisek L, et al. Towards personalized therapy for multiple sclerosis: prediction of individual treatment response. Brain 2017;140: 2426-2443.

57. Sormani MP, Bruzzi P. Can we measure long-term treatment effects in multiple sclerosis? Nat Rev Neurol 2015;11:176-182.

58. Kalincik T, Jokubaitis V, Spelman T, et al. Cladribine versus fingolimod, natalizumab and interferon $\beta$ for multiple sclerosis. Mult Scler J Epub 2017 Aug 1. doi: 10.1177/ 1352458517728812

59. Soelberg Sorensen P, Sellebjerg F. Neurofilament in CSF-A biomarker of disease activity and long-term prognosis in multiple sclerosis. Mult Scler 2016;22:1112-1113.

60. Kuhle J, Nourbakhsh B, Grant D, et al. Serum neurofilament is associated with progression of brain atrophy and disability in early MS. Neurology 2017;88:826-831.

61. Sormani MP, Pardini M. Assessing repair in multiple sclerosis: outcomes for phase II clinical trials. Neurotherapeutics 2017;14:924-933.

62. Cadavid D, Balcer L, Galetta S, et al. Safety and efficacy of opicinumab in acute optic neuritis (RENEW): a randomised, placebo-controlled, phase 2 trial. Lancet Neuro 2017;16:189-199.

63. Green AJ, Gelfand JM, Cree BA, et al. Clemastine fumarate as a remyelinating therapy for multiple sclerosis (ReBUILD): a randomised, controlled, double-blind, crossover trial. Lancet 2017;390:2481-2489.

64. Plemel JR, Liu WQ, Yong VW. Remyelination therapies: a new direction and challenge in multiple sclerosis. Nat Rev Drug Discov 2017;16:617-634 


\section{Neurology}

\section{Comment on 2018 American Academy of Neurology guidelines on disease-modifying therapies in MS}

John R. Corboy, Brian G. Weinshenker and Dean M. Wingerchuk Neurology 2018;90;1106-1112 Published Online before print April 23, 2018

DOI 10.1212/WNL.0000000000005574

\section{This information is current as of April 23, 2018}

\section{Updated Information \& Services}

References

Citations

Subspecialty Collections

Permissions \& Licensing

Reprints including high resolution figures, can be found at: http://n.neurology.org/content/90/24/1106.full

This article cites 55 articles, 15 of which you can access for free at: http://n.neurology.org/content/90/24/1106.full\#ref-list-1

This article has been cited by 2 HighWire-hosted articles: http://n.neurology.org/content/90/24/1106.full\#\#otherarticles

This article, along with others on similar topics, appears in the following collection(s):

\section{All Clinical trials}

http://n.neurology.org/cgi/collection/all_clinical_trials

Multiple sclerosis

http://n.neurology.org/cgi/collection/multiple_sclerosis

Information about reproducing this article in parts (figures,tables) or in its entirety can be found online at:

http://www.neurology.org/about/about_the_journal\#permissions

Information about ordering reprints can be found online:

http://n.neurology.org/subscribers/advertise

Neurology ${ }^{\circledR}$ is the official journal of the American Academy of Neurology. Published continuously since 1951, it is now a weekly with 48 issues per year. Copyright @ 2018 American Academy of Neurology. All rights reserved. Print ISSN: 0028-3878. Online ISSN: 1526-632X.

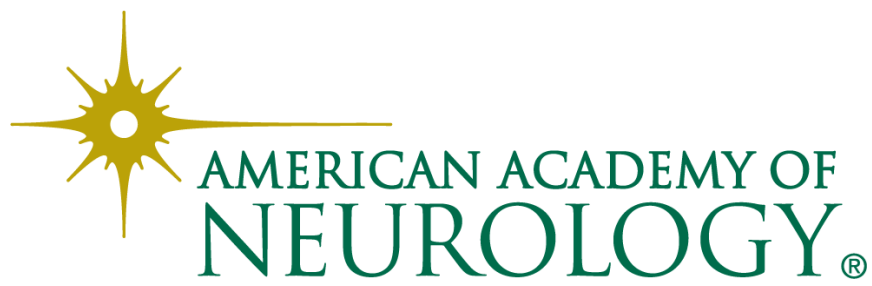

